\section{El Programa de Medicina Transfusional de Cuba}

\author{
José M. Ballester Santovenia ${ }^{1}$
}

Palabras clave: medicina transfusional, Cuba.

1 Instituto de Hematología e Inmunología, La Habana, Cuba. Dirección postal: Instituto de Hematología e Inmunología, Apartado Postal 8070, Ciudad de La Habana, Cuba. Tel.: (537) 578269. Fax: (537) 442334. Correo electrónico: ihidir@hemato.sld.cu.
La República de Cuba, situada en la parte occidental del mar Caribe, es un archipiélago constituido por más de 4200 islas e islotes, entre los cuales destacan Cuba y la Isla de la Juventud. Su superficie total es de $110860 \mathrm{~km}^{2}$ y la población en diciembre del 2002 era de 11308000 habitantes. El país está organizado política y administrativamente en 14 provincias y un municipio especial.

La Constitución de la República garantiza el carácter gratuito y establece la equidad de los servicios de salud, mientras que la Ley 41 de 1983 precisa, entre otras disposiciones, que la donación de sangre debe ser voluntaria y altruista.

\section{ORGANIZACIÓN DEL SISTEMA NACIONAL DE SALUD}

En Cuba hay una red de instituciones para la atención integral de la población —comprende 270 hospitales, 440 policlínicas, 13 institutos de investigación y 6 centros de atención cardiovascular-, las cuales están dedicadas tanto a actividades de prevención, diagnóstico y tratamiento como a la rehabilitación. En su conjunto, el Sistema Nacional de Salud (SNS) cuenta con 58714 camas para la asistencia médica de pacientes hospitalizados, independientemente de las camas de asistencia social, y con 64683 médicos, además de personal de enfermería con y sin licenciatura, técnicos de la salud, profesionales universitarios (bioquímicos, biólogos, químicos) y técnicos de nivel medio (1). En varias de estas instituciones se realizan procedimientos médicos y quirúrgicos complejos, tales como transplantes de órganos y tejidos y cirugía cardiovascular, que requieren el respaldo de grandes cantidades de sangre y derivados sanguíneos para transfusión. Todos los hospitales tienen un comité fármaco-terapéutico que vela por el uso racional de los medicamentos, así como de la sangre y sus componentes.

\section{Subsistema de medicina transfusional}

El Programa de Medicina Transfusional de Cuba es un subsistema del SNS que fue establecido para garantizar la obtención y administración de sangre segura para todas las personas que la necesiten. Está integrado por la Comisión Nacional de 
Medicina Transfusional, el Instituto de Hematología e Inmunología, 37 servicios clínicos, 44 bancos de sangre, 120 centros de extracción fijos, 19 unidades móviles, 37 laboratorios para la certificación de la sangre y servicios de transfusiones en los principales hospitales. También se dispone de un laboratorio para el fraccionamiento del plasma y la obtención de albúmina y gammaglobulinas; un laboratorio para la producción de interferón leucocitario y factor de transferencia; y dos laboratorios para la producción de reactivos utilizados para la hemoclasificación y la detección de sangre infectada. El programa cuenta con suficiente personal capacitado: médicos hematólogos, inmunólogos, especialistas en laboratorio clínico y en medicina general integral, así como con otros profesionales que no son médicos, entre ellos bioquímicos, biólogos, licenciados en enfermería y otros técnicos de nivel medio y superior (cuadro 1). El Instituto de Hematología e Inmunología, como entidad especializada, desempeña un papel importante en este subsistema y coordina varios programas relacionados con la obtención y el control de la sangre para transfusión (cuadro 2).

CUADRO 1. Recursos humanos vinculados al subsistema de medicina transfusionala ${ }^{a}$. La Habana, Cuba

\begin{tabular}{lr}
\hline \multicolumn{1}{c}{ Tipo de profesional } & Cantidad \\
\hline Médicos & 149 \\
Profesionales que no son médicos & 190 \\
Licenciados en enfermería & 61 \\
Técnicos & 865 \\
Personal de enfermería & 124 \\
Personal administrativo y de servicios & 400 \\
Total & 1789 \\
\hline a Personal que presta servicios en bancos de sangre, centros de extracción y en \\
el Instituto de Hematología e Inmunología. No se incluye el de los servicios de \\
transfusiones.
\end{tabular}

CUADRO 2. Programas coordinados por el Instituto de Hematología e Inmunología. La Habana, Cuba

- Medicina transfusional

- Inmunodiagnóstico

- Leucemias

- Anemia drepanocítica

- Hemofilias

- Anemias nutricionales

- Transplante de médula ósea

- Enfermedad hemolítica del feto y el recién nacido

- Medicina natural y tradicional. Homeopatía

- Medicina de desastres
En el año 2002 se realizaron en Cuba 563204 donaciones voluntarias de sangre y plasma ( 1 por cada 20 habitantes). De ellas, 29000 (5,1\%) pertenecían al programa de donación por aféresis, que se encarga de recolectar plasma normal y plasma con anticuerpos contra los agentes causales del tétanos y de la hepatitis $B$ y contra el antígeno $D\left(R h_{o}\right)$.

Todas las donaciones se someten individualmente a un tamizaje para la detección de anticuerpos contra VIH-1 y 2, contra los virus de las hepatitis B y C y contra el agente causal de la sífilis (prueba VDRL). Como resultado de este tamizaje, en 2002 se descartó $0,012 \%$ de la sangre donada por haber resultado positiva al VIH-1 y $2 ; 0,60 \%$ por positividad al virus de la hepatitis $\mathrm{B} ; 0,71 \%$ por positividad al virus de la hepatitis $C$ y $1,8 \%$ por positividad en la prueba de VDRL. En general, el volumen de sangre descartada ese año por haber mostrado reactividad a las pruebas de tamizaje serológico, caducidad (glóbulos rojos fundamentalmente) o por otras causas fue del 9,8\%. Ese año se realizaron 445898 transfusiones de sangre y de hemoderivados (cuadro 3) $(2,3)$. La diferencia entre la cifra de donaciones y la de transfusiones se debe a las bajas por los resultados del tamizaje y al plasma para fraccionamiento entregado a la planta de hemoderivados.

En Cuba no hay casos de enfermedad de Chagas. En un estudio realizado en 1991 en colaboración con la Universidad de Kumamoto, Japón, se encontró que la prevalencia de HTLV-I y II en Cuba era muy baja $(4,5)$ y se consideró que no era imprescindible en ese momento incluir esas pruebas en el tamizaje de la sangre, teniendo en cuenta, además, las serias dificultades que ha venido enfrentando Cuba, las cuales han obligado a las autoridades sanitarias a establecer prioridades para los tamizajes. No obstante, en la actualidad el Centro de Inmunoensayo y el Laboratorio de Fraccionamiento de Plasma, que son los centros nacionales de producción de reactivos, están luchando por poner en práctica un sis-

CUADRO 3. Transfusiones de sangre y de sus componentes. Cuba, 2002

\begin{tabular}{lr}
\hline Componente & Unidades \\
\hline Sangre total & 1700 \\
Eritrocitos & 276228 \\
Plasma & 74605 \\
Plaquetas & 56069 \\
Crioprecipitado & 37296 \\
$\quad$ Total & 445898 \\
\hline
\end{tabular}


CUADRO 4. Producciones del subsistema de medicina transfusional. Cuba, 2002

- Productos habituales de los bancos de sangre

- Albúmina

- Gammaglobulinas polivalentes y específicas

- Interferón leucocitario alfa

- Factor de transferencia

- Sueros hemoclasificadores polivalentes y monoclonales

- Reactivos para tamizaje de VIH 1 y 2 , hepatitis B, hepatitis C y sífilis. $^{a}$

a Un sistema para el tamizaje de HTLV I y II está en proceso de desarrollo.

tema de diagnóstico con anticuerpos detectores de HTLV-I y II de producción nacional, medida que posibilitará incorporar estas pruebas al tamizaje individual de los donantes de sangre.

A los laboratorios dedicados a la producción de hemoderivados y reactivos se les suministró plasma normal e hiperinmune y masa leucocitaria para la producción de albúmina, gammaglobulinas, interferón, factor de transferencia y reactivos para la hemoclasificación (cuadro 4) (6-10).

\section{Comisión Nacional de Medicina Transfusional}

El Ministerio de Salud Pública de la República de Cuba (MINSAP) coordina este programa por mediación de la Comisión Nacional de Medicina Transfusional (CNMT), cuyas principales funciones son:

- Garantizar la autosuficiencia del abastecimiento de sangre y de sus componentes con la máxima seguridad y calidad posibles

- Velar por el cumplimiento de los principios éticos, de acuerdo con lo estipulado en el Código de Ética de la Sociedad Internacional de Transfusión de Sangre y en las Regulaciones Deontológicas del MINSAP.

- Controlar la aplicación de todas las normas médicas y técnicas relacionadas con la hemoterapia

- Promover la investigación científica y el desarrollo tecnológico en materia de hemoterapia

- Desarrollar nuevos productos para uso clínico y de laboratorio

Las responsabilidades de los integrantes de la CNMT están distribuidas de la siguiente manera:

MINSAP. Controla, como organismo rector, todas las actividades relacionadas con la garantía de la salud de la población y su financiamiento.
Instituto de Hematología e Inmunología. Norma los elementos metodológicos y científico-técnicos, así como la producción de componentes y sueros hemoclasificadores en los bancos de sangre.

Laboratorio de Fraccionamiento de Plasma. Produce albúmina, gammaglobulinas polivalentes y específicas y está desarrollando la producción de factores VIII y IX de la coagulación.

Centro de Inmunoensayo. Produce los reactivos necesarios para el tamizaje de la sangre mediante una tecnología de su propia elaboración: el sistema ultramicroanalítico (SUMA).

Centro de Inmunología Molecular. Produce y desarrolla anticuerpos monoclonales.

Centro de Investigaciones Biológicas. Produce interferón leucocitario y factor de transferencia.

Laboratorio Nacional de Control del VIH. Como centro de referencia, es la entidad nacional responsable del control externo del tamizaje de los virus de la inmunodeficiencia humana.

Sociedad Cubana de la Cruz Roja. Participa en la promoción de la donación de sangre y en la organización de la hemoterapia en situaciones de desastre.

Centro para el Control Estatal de la Calidad de los Medicamentos. Controla los procedimientos normados para la producción de componentes y reactivos.

Comités de Defensa de la Revolución (CDR). Constituyen una organización social comunitaria que participa en y apoya muchas actividades de salud pública dentro de cada comunidad y que promueve entre sus miembros, junto con otras organizaciones y los médicos de familia, la donación voluntaria y altruista de sangre.

\section{LA DONACIÓN DE SANGRE EN CUBA}

La organización de la donación voluntaria de sangre en Cuba se inició en 1962 y las aportaciones han aumentado sistemáticamente. Aunque ya en 1990 se superaban las 500000 donaciones anuales, una parte era para reposición. No fue hasta 1997 que se logró alcanzar la meta propuesta por la OMS de una donación voluntaria de sangre por cada 20 habitantes (figura 1). Cada año se realizan diversas actividades para motivar a los donantes. Un ejemplo es el de la Semana del Donante Voluntario, que 
FIGURA 1. Donaciones voluntarias de sangre, 1962-2000

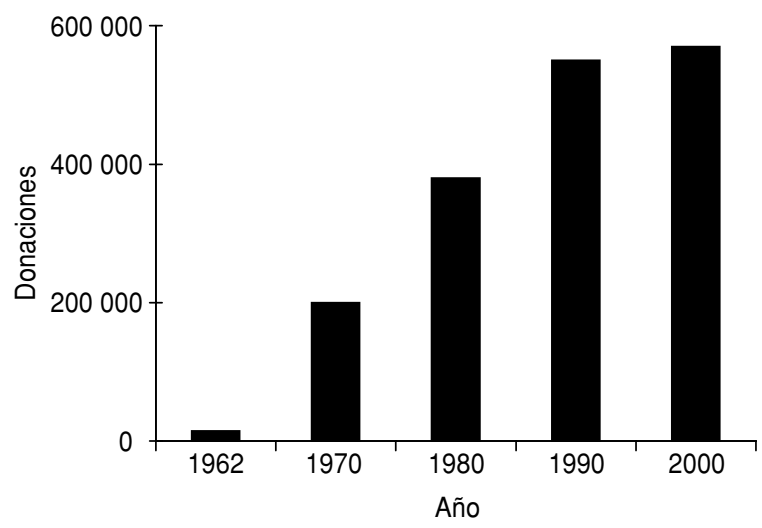

se celebra en el marco de las celebraciones por el natalicio del Dr. Carlos J. Finlay (3 de diciembre). Durante estas jornadas se realizan actos sociales y culturales en todos los municipios del país, y la comunidad rinde homenaje a los donantes más destacados mediante diplomas, sellos distintivos y otros reconocimientos.

No obstante la situación financiera adversa que ha enfrentado Cuba durante la última década, se ha logrado garantizar sangre segura a la población. Esto lo evidencia el hecho de que desde 1985 solamente se han notificado infecciones postransfusionales por VIH en tres enfermos de hemofilia.

\section{OBJETIVOS INMEDIATOS DEL PROGRAMA DE MEDICINA TRANSFUSIONAL}

El Programa de Medicina Transfusional de Cuba tiene como objetivos inmediatos los siguientes (11):

- Acabar de organizar el Registro de Donantes Habituales e informatizar la red de bancos de sangre

- Incrementar la obtención de plasma para fraccionamiento mediante plasmaféresis

- Establecer el sistema de hemovigilancia en todo el país
- Desarrollar los sistemas de control de calidad en todos los servicios de sangre según las recomendaciones de la OPS $(12,13)$

- Iniciar la producción de concentrados de los factores VIII y IX de la coagulación

- Incorporar nuevas tecnologías para mejorar la seguridad de la sangre (14)

- Aplicar las pautas relacionadas con la seguridad de la sangre según la OPS hasta el año 2003 (15).

Agradecimientos. Se le agradece a Walkyria Cao Fonticoba su valiosa colaboración en la preparación de este trabajo.

\section{SYNOPSIS}

\section{The Transfusion Medicine Program in Cuba}

Cuba's Transfusion Medicine Program (TMP) is a subsystem of the country's National Health System. The TMP's objective is to ensure hemotherapy with blood that is safe and sufficient for all the individuals who need it. The TMP subsystem is made up of the National Commission on Transfusion Medicine, the Institute of Hematology and Immunology, 37 clinical services, 44 blood banks, 120 collection centers, 19 mobile units, and 37 blood certification laboratories. Additional facilities include a laboratory for plasma separation, a laboratory that produces leukocyte interferon and transfer factor, and two laboratories that produce reagents for blood classification and blood diagnosis systems.

In Cuba, blood donation is voluntary. Since 1997 approximately 5\% of the population per year has donated blood, thus meeting the goal recommended by the Pan American Health Organization of one voluntary blood donation annually for every 20 persons. During 2002, 563204 blood donations were received, and there were 445898 transfusions of blood or blood components. All donations are individually screened for HIV 1 and 2, hepatitis B, hepatitis C, and syphilis, thus meeting the country's current regulations. In 2002 these screening measures led to discarding, respectively, $0.12 \%, 0.60 \%, 0.71 \%$, and $1.8 \%$ of the blood donations. Although the prevalence of human T-cell lymphotropic virus I and II in Cuba is very low, this test will soon be added to the screening process. 


\title{
REFERENCIAS
}

1. National Health Statistics Bureau. Ministry of Public Health. Annual Health Statistics Report. La Habana: Ministerio de Salud Pública; 2001.

2. López MR, Cortina L, González T, Salgado $\mathrm{O}$. Evaluación de las indicaciones de plasma fresco congelado en el Hospital General Docente "Enrique Cabrera", de la Ciudad de La Habana. Rev Argentina Transfus 2001;27:4

3. Rubio R, Cárdenas E, Pérez LM, Halley B. Comportamiento de la hemoterapia en la Ciudad de La Habana, 1992-1997. Rev Cubana Hematol Inmunol Hemoter 1998;14:186-188.

4. Hernández P, Rivero R, Ballester JM, Navea L, Matutes E, Catovski E, et al. Very low seroprevalence of HTLV-I/II in Cuba: antibodies in blood donors and in hematological and non-hematological patients. Vox Sang 1991;61:277-278.

5. Rivero $R$, Hernández $P$, Suárez $O$ Hidalgo-Gato R, Navea, LM, Yamaguchi K, Ballester JM. Detección de anticuerpos contra el virus linfotrópico de la célula T HTLV-I/II en pacientes cuba- nos con insuficiencia renal crónica en hemodiálisis. Sangre 1992;37:205-206.

6. Rivero R, Suárez L, Bencomo A. Características serológicas y análisis inmuhohistológico del anticuerpo monoclonal IHI-15 anti-A: resultados de su evaluación en Nantes. Rev Cubana Hematol Inmunol Hemoter 1998;14:158166.

7. Rivero R, Suárez L, Bencomo A, González R, Martínez M, Tormo B, Rodríguez T. Ensayo de terreno de los reactivos hemoclasificadores monoclonales HemoCIM anti-A y Hemo-CIM anti-B como productos terminados. Rev Cubana Hematol Inmunol Hemoter 1998;14:167175.

8. Suárez L, Rivero R, Pérez N, Bencomo A, Torres F, Martínez M, et al. Reactivo hemoclasificador monoclonal cubano Hemo-CIM anti-B. Estudio de estabilidad. Rev Cubana Hematol Inmunol Hemoter 1999;15:25-29.

9. Bencomo A, Alfonso Y, Rivero R. Reactivity of anti-D monoclonal antibodies with $\mathrm{D}$ variants in red blood cells from black Cuban persons. Transfus Clin Biol 2001;8:246s.

10. Alfonso Y, Bencomo A, Díaz M, Rivero R. Serology of antibodies to $A B O$ and other carbohydrate antigens. Transfus Clin Biol 2001;8:251s.

11. Cuba, Ministerio de Salud Pública. Programa de Desarrollo 2000, hematología. La Habana: MINSAP; 1988.

12. Organización Panamericana de la Salud. Estándares de trabajo para bancos de sangre (2. a versión). OPS, División de Desarrollo de Sistemas y Servicios de Salud. Washington, D.C.: 1999. (Serie Medicamentos Esenciales y Tecnología).

13. Organización Panamericana de la Salud. Nuevos rumbos para la salud en las Américas. Informe Cuadrienal del Director. Edición del Centenario. Washington, D.C.; 2002.

14. Hutt E. Donor safety: the safety of modern plasma products. HQ Haemophilia Quart (UK) 2002;(2):13.

15. World Health Organization. Blood safety and clinical technology: strategy 20002003. Geneva: WHO; 2001.

\section{Annual Conference The Global Health Council}

\author{
Dates: $\quad$ 27-30 May 2003 \\ Location: Omni Shoreham Hotel \\ Washington, D.C. \\ United States of America
}

The theme of the 30th Annual Conference of the Global Health Council (GHC) is "Our Future on Common Ground - Health and the Environment." The conference will examine how the physical environment, the economic environment, and the social environment all exert a profound influence on health, with many of the world's health disparities deriving from underlying environmental conditions.

The conference will have plenary sessions, with well-known speakers addressing the general themes of the meeting. There will also be numerous concurrent panel sessions in which three or four presenters will each present on a specific topic, followed by a question-and-answer period. Among the general subject areas of the panel sessions are physical environmental health, infectious diseases, reproductive and maternal health, health systems policy and research, social environmental health, child and adolescent health, and HIV/AIDS. There will also be a large exhibition, with booth displays by more than 70 organizations and groups concerned with international health.

The conference is intended for health care providers, community organizers, program managers, policymakers, researchers, and health advocates. The conference registration fee for GHC members is US\$ 325 if paid by 9 May, and US $\$ 400$ after that date. The respective fees for persons who are not GHC members are US\$ 455 and US\$ 555. There are also special reduced registration fees for students.

\author{
Information: \\ Global Health Council \\ 1701 K Street, N.W., Suite 600 \\ Washington, D.C. 20006-1503, United States of America \\ Telephone: (202) 833-5900 • Fax: (202) 833-0075 \\ Email: ghc@globalhealth.org \\ Internet: http://www.globalhealth.org
}

